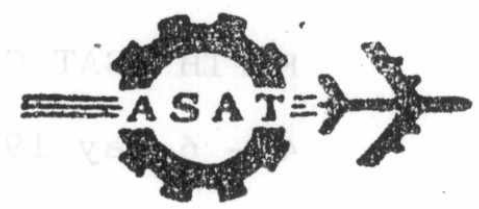

\title{
STABILITY ANALYSIS OF PASSIVE CONTINUOUS-WAVE DOPPLER SEEKERS
}

\author{
MOHSEN SALAH ALY*
}

\begin{abstract}
Passive monopulse CW-doppler seeker plays the main role in the guidance and control of semiactive radar homing guided missiles. The most common seeker configuration involves frequency-locked loop (FLL) connected with a phase-locked loop (PLL) through a common phase detector and a local voltage controlled oscillator (LVCO). In this paper, analysis of the operation of such seeker system is presented. The control equations are derived wherefrom the system transfer function is obtained. Stability analysis is also carried out. It is shown that the presence of low-pass filter at the input of the LVCO is essential for system stability. Moreover, the filter time constants should be carefully adjusted to ensure stable operation.
\end{abstract}

\section{INTRODUCTION}

In semiactive guided missiles, the guidance commands are generated on the missile board. A monopulse passive tracking radar placed at the missile nose is designed to accurately track the illuminated target. This radar is known as seeker. The seeker circuits generate the control signals that steer the missile in space. The seeker employs two antennas to perform its function; the front target tracking antenna and the rear reference antenna.

The target front antenna is designed to detect the reflected echo from the target being engaged. The rear antenna; however, is used to detect the reference signal from the parent illuminator. Fig. 1 illustrates the role of each antenna in the guidance process. Recent seeker systems involve a PLL to keep the LVCO locked on the parent illuminator signal. In the mean time, a FLL is often used to track the doppler frequency changes in the target echo return. Both loops are involved instanteneously in the adjustment of the LVCO frequency. Fig. 2 shows the block diagram of the system being considered.

The performance of the FLL and the PLL had been extensively analyzed long time ago [1]-[4]. The control equations and the stability analysis of both types of loops had been established as well. In addition, the behavior of the FLL and the PLL in the presence of two input signals have been a matter of discussion in the last two decades [5]-[7]. The jump phenomenon that occurs in the presence of two input signals had been investigated by many researchers. The importance of the subject is attributed to the role played by the FLL and the PLL in adjusting the operation of the missile seeker and hence; in controlling the guided missile during flight.

* Ph.D., Guidance Department, M.T.C., Cairo, Egypt. 
Despite the large effort dedicated in analyzing the performance of the FLL and the PLL individually, very little effort has been done in the investigation of the combined loops as shown in Fig. 2. The purpose of this paper is to investigate the operation and performance of the combined loops shown in Fig. 2. The system elements are assumed to be linear, i.e; the frequency of the different signals across the loops are near their correct values. The system response to the frequency variations of the front and rear received signals is investigated. Routh-Hurwitez stability analysis is applied to the system transfer function. It is shown that a low-pass filter at the input of the LVCO is essential for the system stability; a requirement which is not necessary for the stability of the conventional FLL or PLL. A commonly used first order low-pass filter is employed. The stability conditions derived impose strict requirements on the filter time constants in terms of the FLL time constant and the PLL gain.

\section{SYSTEM OPERATION}

The system of concern is shown in Fig. 1. The frequency of the signal received by the front antenna is

$$
f_{f}=f_{o}+2 f_{t}+f_{m}
$$

where $f_{o}$ is the illuminator frequency, $f_{t}$ and $f_{m}$ are the target and missile doppler frequencies; respectively, and are given by

$$
f_{t}=\frac{v_{t}}{\lambda_{o}}
$$

and

$$
f_{m}=\frac{v_{m}}{\lambda_{o}}
$$

where $\lambda_{o}$ is the wavelength of the illuminator signal, $v_{t}$ and $v_{m}$ are the target and missile radial speeds; respectively. The rear antenna; however, receives the illuminator signal directly. Thus, the frequency of the signal received by the rear antenna will be

$$
f_{r}=f_{o}-f_{m}
$$

As shown in Fig. 2, the signal received by the rear antenna is mixed with the LVCO output. The phase of the resultant signal (rear IF) is compared with the phase of another signal generated by the front FLL. The phase detector output is filtered and then used to control the frequency of the LVCO. In the mean time, the LVCO output is mixed with the front received signal in the mixer M1. The output of M1 (first IF) is then amplified and filtered. Practically, the frequency band of the first IF is not suitable for further processing. Thus, the first IF is mixed in M2 with a locally generated sinusoidal signal. The frequency of M2 output (second IF) is in the low frequency band where frequency discrimination can be performed efficiently. After discrimination, integration, and amplification, the output signal is used to control the frequency of a voltage controlled oscillator (VCO). The output 
of the VCO is then mixed with another locally generated sinusoidal signal in M3. The phase of M3 output is compared with the rear IF phase as mentioned before.

\section{FREQUECY-LOCK-LOOP OPERATION}

The frequency of the signal at the output of the mixer M1 is

$$
f_{1}=f_{l o}-f_{f}
$$

where $f_{l o}$ is the frequency of the LVCO output. Usually the frequency $f_{1}$ is in the megahertz band which is not suitable for further processing. Thus, further reduction is made in the mixer M2 by mixing the output of the band-pass filter (BPF) with a sinusoidal signal of frequency $f_{o 1}$. The frequency of the mixer M2 output is

$$
f_{2}=f_{1}-f_{o 1}
$$

The output of the mixer M2 is fed to a frequency discriminator with frequency center at $f_{d i s}$ and gain $k_{d}$. Thus, its output voltage is

$$
V_{d}=K_{d}\left(f_{l o}-f_{f}-f_{o 1}-f_{d i s}\right) .
$$

The central frequency of the first IF bandpass filter is denoted by $f_{x t l}$ and is related to $f_{o 1}$ and $f_{d i s}$ by

$$
f_{x t l}=f_{o 1}+f_{d i s} .
$$

The frequency discriminator output is integrated, amplified, and then used to drive a VCO with gain $K_{v}$. The VCO output frequency will thus be

$$
f_{3}=\frac{V_{d} K_{a} K_{v}}{T^{\prime} s}+f_{o 3}
$$

where $T^{\prime}$ and $K_{a}$ are the integrator time constant and the amplifier gain; respectively. $f_{o 3}$ is the base frequency of the VCO ( the VCO output frequency for zero input voltage). The $\mathrm{VCO}$ output frequency is stepped up in the mixer M3 as

$$
f_{4}=\frac{V_{d} K_{a} K_{v}}{T^{\prime} s}+f_{o 3}+f_{o 2},
$$

where $f_{o 2}$ is the frequency of a locally generated sinusoidal signal. It is noted that the following relation holds

$$
f_{x t l}=f_{o 2}+f_{o 3} .
$$

Thus, $f_{4}$ can be rewritten as

$$
f_{4}=f_{x t l}+\frac{1}{T s}\left(f_{l o}-f_{f}-f_{x t l}\right),
$$


where $T$ defines the time constant of the FLL and is given by

$$
T=\frac{T^{\prime}}{K_{a} K_{v} K_{d}}
$$

\section{PHASE-LOCK-LOOP OPERATION}

After mixing the received signal from the rear antenna in M4 with the LVCO output and filtering out the higher frequency components, the frequency $f_{5}$ of the phase detector input will be

$$
f_{5}=f_{l o}-f_{r}=f_{l o}-f_{o}+f_{m} .
$$

The phase difference between the phase detector inputs is detected in the phase detector and can be written as

$$
V_{p d}=K_{p d}\left(\phi_{5}-\phi_{4}\right)=\frac{2 \pi K_{p d}}{s}\left(f_{5}-f_{4}\right)
$$

In view of equations 11,12 , and 13 , the LVCO frequency can be written as

$$
f_{l o}=f_{o}+f_{x t l}+K_{l o} F(s) V_{p d}
$$

where $f_{o}+f_{x t l}$ is the base frequency of the LVCO and $F(s)$ is the low-pass filter (LPF) transfer function.

\section{SYSTEM TRANSFER FUNCTION}

Eq. 14 can be rearranged as

$$
f_{l o}^{\prime}=\frac{1}{s T_{p l l}} F(s)\left[\left(f_{l o}^{\prime}+f_{o}-f_{r}\right)-\frac{1}{T s}\left(f_{l o}^{\prime}+f_{o}-f_{f}\right)\right]
$$

where $T_{p l l}$ and $f_{l o}^{\prime}$ are given by

$$
T_{p l l}=\frac{1}{K}=\frac{1}{2 \pi K_{p d} K_{l o}}
$$

and

$$
f_{l o}^{\prime}=f_{l o}-\left(f_{o}+f_{x t l}\right)
$$

It is noted that $T_{p l l}$ is the inverse of the open PLL gain $K$. Rearrangement of Eq. 15 leads to

$$
f_{l o}^{\prime}=G(s)\left[s T\left(f_{o}-f_{r}\right)+\left(f_{f}-f_{o}\right)\right]
$$


where $G(s)$ is given by

$$
G(s)=\frac{F(s)}{s^{2} T T_{p l l}-s T F(s)+F(s)} .
$$

Equation 16 represents the control equation of the FLL and the PLL connected as shown in Fig. 2. It is obvious that the filter is necessary for the system stability. In the absence of this filter $(F(s)=1)$, the two loops will not be able to adjust the LVCO frequency to follow the frequency variations in $\left(f_{o}-f_{r}\right)$ and $\left(f_{f}-f_{o}\right)$.

\section{SYSTEM STABILITY}

The system under consideration is a two-input, single-output system. The most frequently used LPF is given by [1]

$$
F(s)=\frac{1+\tau_{2} s}{1+\tau_{1} s}
$$

with $\tau_{2}$ being smaller than $\tau_{1}$. By employing this filter, the transfer function $G(s)$ can be rewritten as

$$
G(s)=\frac{1+\tau_{2} s}{T T_{p l l} \tau_{1} s^{3}+T\left(T_{p l l}-\tau_{2}\right) s^{2}+\left(\tau_{2}-T\right) s+1} .
$$

Application of Routh-Hurwitz stability test leads to the following stability conditions

$$
\frac{1}{K}>\tau_{2}>T
$$

and

$$
\tau_{1}<\left(1-K \tau_{2}\right)\left(\tau_{2}-T\right)
$$

It is known that the PLL working alone is unconditionally stable. However, for the present system, the two conditions given by Eq. 20 are necessary for stable operation. Inspection of Eq. 20 shows that the PLL gain should not exceed a limit value given by $\frac{1}{\tau_{2}}$. Moreover, the time constant of the FLL should not exceed the filter time constant $\tau_{2}$. The fact that the natural frequency of the PLL is inversely proportional to $\tau_{1}$ [1], which should not exceed a limit value given by Eq. 20, indicates that the tracking speed of the PLL should not exceed a limit value as well.

In the steady state; however, the LVCO frequency is merely determined by the second term of Eq. 16 as:

$$
f_{l o s s}=f_{x t l}+2 f_{t}-f_{m} .
$$

It is seen from Eq. 21 that the mixer M1 steady state output frequency is equal $f_{x t l}$ but due to the dynamic nature of the frequencies of the input signals, the frequency of M1 output can not be held at $f_{x t l}$. However, any deviation form $f_{x t l}$ is detected by the frequency discriminator which adjusts the VCO frequency so that $f_{4}$ matches $f_{5}$. Any mismatch between $f_{4}$ and $f_{5}$ is detected by the phase detector which tune the LVCO so that balance is achieved. 


\section{CONCLUSIONS AND FUTURE RESEARCH}

The operation of the FLL-PLL combination is investigated. The system transfer function is derived. Routh-Hurwitez stability analysis is carried out. It is found that a low-passfilter has to be inserted at the input of the LVCO for system stability. In addition, the filter time constants have to be carefully adjusted to obtain stable operation. The relative stability margins and the system response to more than one input signal from the front antenna are points of increasing interest and need more investigations.

\section{REFERENCES}

[1] Blanchard, A.: " Phase Lock Loops". (J. Wiley, New York, 1976)

[2] Gardner, F. M.: " Phase Lock Loops". (J. Wiley, New York, 1979)

[3] Lindsey, W. C. and Simon, M. K. (Eds.): "Phase Locked Loops and Their Applications". (IEEE Press, New York, 1978)

[4] Klapper, J. and Frankle, J. T.: "Phase Locked and Frequency Feedback Systems". (Academic Press, New York, 1972)

[5] Kliger, I. and Olenberger, C.: "Phase Lock Loop Jump Phenomenon In the Presence of Two Signals" , IEEE Trans. Aerosp. Electron. Syst., vol. AES-12, pp. 55-64, January 1976:

[6] Olenberger, C.: "Effects of Automatic Gain Control on Phase Lock Loop Behavior in the Presence of Interference" , IEEE Trans. Aerosp. Electron. Syst., vol. AES-14, pp. 803-805, November 1977.

[7] Bar-Ness, Y. and Bunin, H.: "Interference in Frequency Locked Doppler Tracking Loops" , IEEE Trans. Aerosp. Electron. Syst., vol. AES-20, pp. 574-582, Sept. 1984.

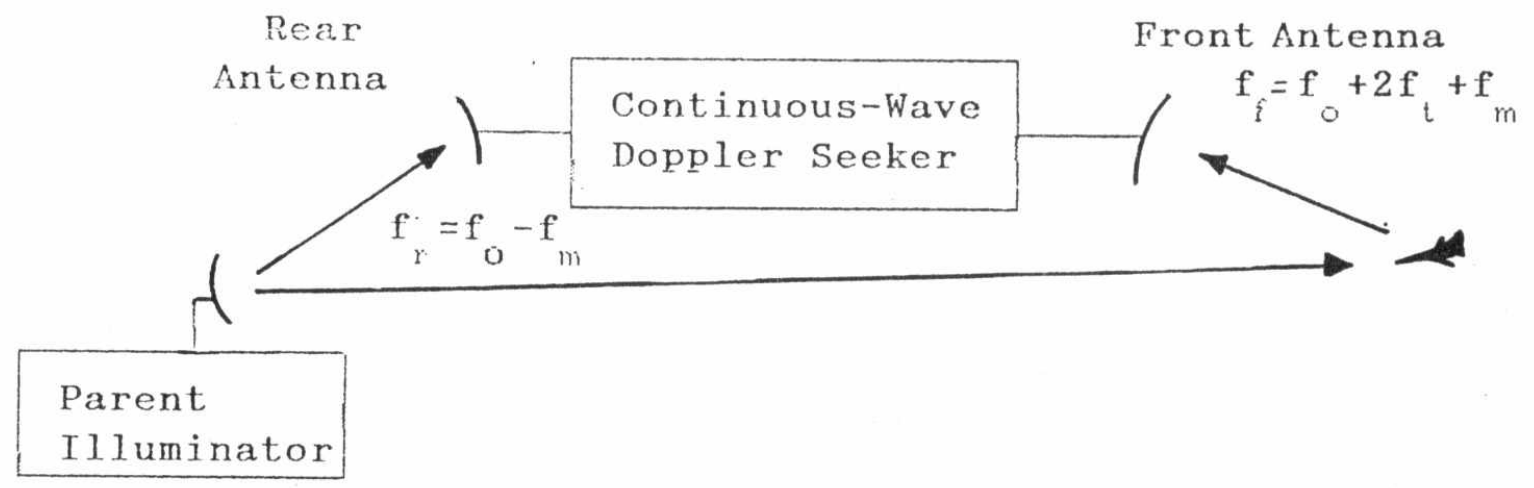

Figure 1: Operation Principle of Continuous-Wave Doppler Seeker 


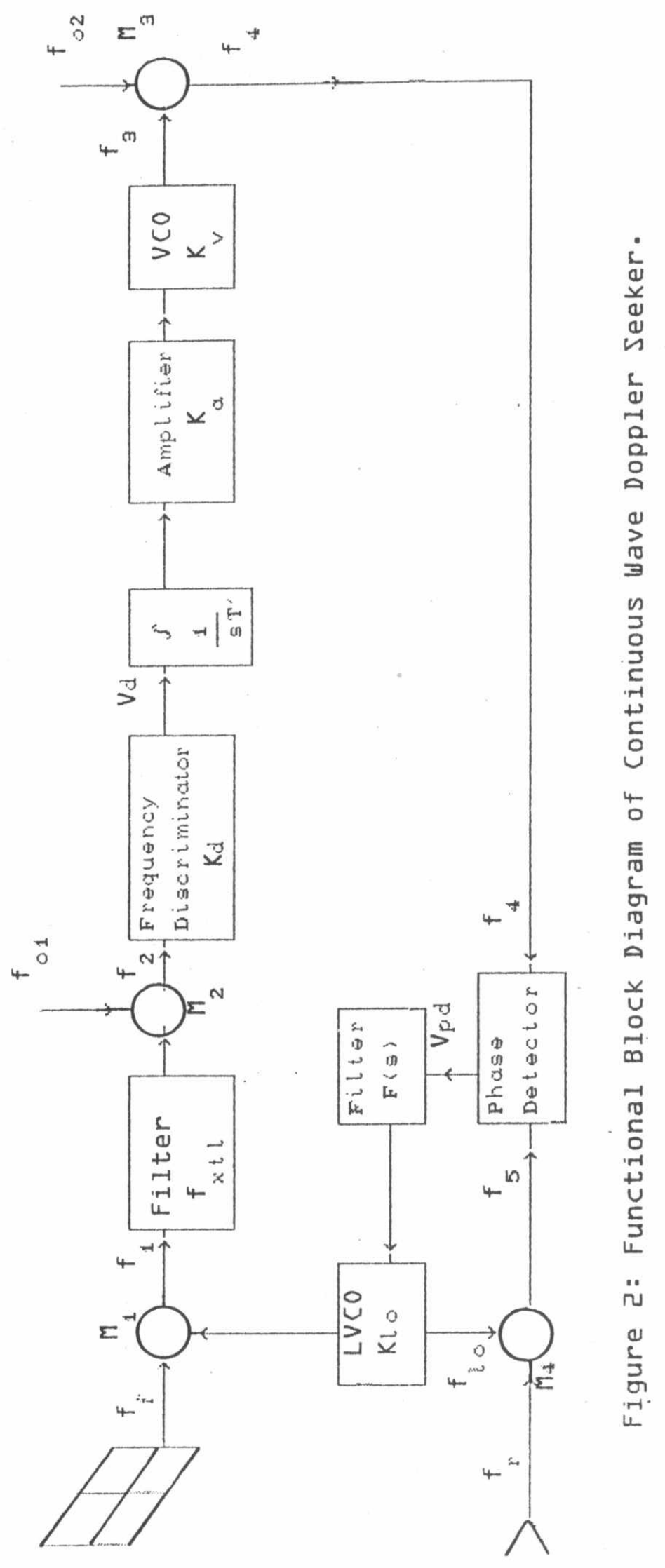

